\title{
Publisher Correction: Live imaging of stem cells in the germarium of the Drosophila ovary using a reusable gas-permeable imaging chamber
}

Amy Reilein, Elisa Cimetta, Nina M. Tandon, Daniel Kalderon, Gordana Vunjak-Novakovic (D)

Correction to: Nature Protocols https://doi.org/10.1038/s41596-018-0054-1, published online 22 October 2018

The version of this paper originally published contained an incorrect unit abbreviation in Step 21: " $0.20 \mathrm{~g} / \mathrm{mL}$ " should have been " $0.20 \mathrm{mg} / \mathrm{mL}$." In addition, the first sentence in Step 33 should have read "Use a second pipette with a cut-off pipette tip to add Matrigel to the center well," instead of "Use a second pipette to cut off the tip of the pipette and add Matrigel to the center well." These errors have been corrected in the PDF and HTML versions of the protocol.

Published online: 15 February 2019

https://doi.org/10.1038/s41596-019-0134-X 